\title{
Profile
}

\section{Shepherd Center}

\author{
D Apple and J Stanton
}

Shepherd Center (Figure 1) is a model of hope. Staff members wear colorful scrubs or casual clothes, making patients feel relaxed and comfortable. Artwork and photographs of the former patients actively involved in pursuits of their choosing grace the walls. Ceilings feature whimsical painted butterflies and hallways are busy with patients moving about in battery-powered wheelchairs navigated by sip-andpuff, head-drive, hand- or chin-controlled mechanisms, all designed to empower them with the freedom of movement and feeling of control.

Shepherd was designed specifically for SCI rehabilitation, so that indoors and out it is totally accessible and exceptionally conducive to patient independence. Everything - wide hallways and doors with lever handles, lowered water fountains and light switches, the auditorium without seats, and the pool with ramps and lifts - is acceessible. Devoted to SCI rehabilitation is a 40-bed floor, plus an 8-bed intensive care unit and a fully equipped therapy gym. Another 32 beds are dedicated to follow-up medical and surgical care, and a distinct 20-bed unit is for the treatment of people with acquired brain injuries. A comprehensive outpatient center provdes a facility for follow-up care. The hospital has a total of five therapy gyms, a fitness center, a cafeteria, inpatient pharmacy and outpatient apothecary, recreation room, outdoor garden and classrooms for eduation and training.

The largest of the nation's 18 model spinal cord injury (SCI) centers, Shepherd consistently sets records for higher numbers of patients discharged to the community versus long term care facilities, higher numbers of patients who are more independent at discharge and lower re-admission rates. Outcomes confirming our position in the industry are reported to the Uniform Data System for Medical Rehabilitation in conjunction with the State University of New York at Buffalo. In comparison to 25 other freestanding rehabilitation hospitals in the Southeast, far more patients from Shepherd returned home and improved functioning than patients discharged from those hospitals.

Correspondence: Judy Stanton, Shepherd Center, 2020 Peachtree Road, NW Atlanta, Georgia 30309, USA
Since 1982, Shepherd has been a model spinal cord injury center, designated by the National Institute on Disability and Rehabilitation Research of the US Department of Education. Model centers play a key role in shaping catastrophic care delivery: providing emergency medical services, acute care, rehabilitative care, psychosocial adjustment, long term follow-up and community reintegration, an injury prevention program, and research. Only 18 model centers were designated in 1995; only 24 since the inception of the model system in 1971. Shepherd Center is Georgia's only model center, one of two in the Southeast. This national recognition carries with it a 5-year, \$1.9 million spinal cord injury research grant.

Since Shepherd's inception, it has earned Joint Commission on Accreditation of Healthcare Organizations (JCAHO) accreditation, the most recent in 1994 for three years. Shepherd has also earned ongoing accreditation from CARF: The Rehabilitation Accreditation Commission, the preeminent standard-setting and accrediting body promoting the delivery of quality rehabilitation services. To be CARF accredited, a Spinal Cord Rehabilitation System of Care must provide a coordinated and integrated continuum of services and should have a minimum of 70 new admissions per year to maintain its identity as a specialized system of care.

Shepherd Center is a specialty hospital. Unlike general rehabilitation facilities, we don't treat patients with hip fractures, strokes, arthritis, musculoskeletal or knee problems. Catastrophic central nervous system injuries make up only about $2 \%$ of an average rehab unit's population. But at Shepherd Center, it's all we do. Every year, Shepherd treats 250 patients with a new spinal cord injury or disease. Since its inception, more than 500 dual diagnosis cases - patients with both SCI and traumatic brain injury - have been treated here.

Specialization means that all our facilities, programming, equipment and technology are designed specifically for spinal cord injured patients. Patients are trained in self-care, addressing issues specific to their needs, from specially-fitted wheelchairs, to bladder management, or assistive technology. Here, spinal cord injury patients identify and interact with others who have similar concerns: patients of the same sex and age, with a similar level and type of injury, whether it's paraplegia, a dual diagnosis of SCI and 


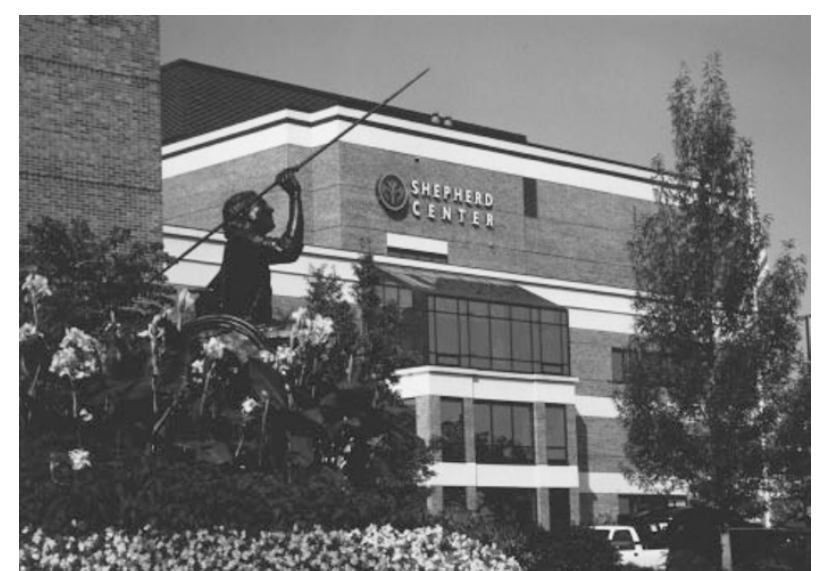

Figure 1 Shepherd Center is located in a lovely area of Atlanta, Georgia. The 100-bed hospital and rehabilitation center is privately owned and not-for-profit

brain injury, tetraplegia or high tetraplegia, including those who are ventilator dependent. And we have more than 20 years experience.

More than $30 \%$ of patients with a spinal cord injury also have a brain injury. Even mild to moderate brain injury can impact on the rehabilitation of SCI patients. Shepherd Center's brain injury unit can treat the most catastrophic, complex brain injuries, known as acquired brain injuries (ABI). Our ABI program treats about 200 patients annually. Treatment teams evaluate the patient's injury to determine placement; care is effectivley coordinated so that dual diagnosis patients have both injuries treated concomitantly.

\section{A continuum of care}

From the point of injury to discharge and follow-up, patients can receive all the services necessary at the appropriate level of care at Shepherd.

\section{Early intervention}

Rehabilitation starts as soon as the patient is able. Patients who begin rehabilitation early in a specialized center experience fewer medical complications, spend less total time in the hospital, and experience higher levels of independence at discharge.

\section{Intensive care (ICU)}

Patients are accepted directly from emergency rooms. Upon transfer, they are evaluated by physicians with extensive experience in catastrophic care, and are placed in intensive care if necessary.

\section{Inpatient rehabilitation}

Once the patient is stable and able to actively participate, full-scale inpatient rehabilitation begins with at least three hours of therapy daily. Physical, occupational and recreational therapies teach mobility, activities of daily living and community reintegration. Skin care, bowel and bladder management are initiated and taught. The psychological readiness of the patient is monitored and addressed, and family and patient education and training begins to teach about the injury, its consequences, and preparation for discharge. The home environment is assessed for accessibility either by a home visit or a review of information with the family. Vocational counselors begin by checking pre-employment history and develop a plan for return to work or school.

\section{Day program}

Patients who become independent of nursing care can continue to receive intensive therapy in the day program. Services are individually tailored to patient needs, and may include occupational, physical and speech therapies, psychology, counseling, education and training, therapeutic recreation and vocational services.

\section{Transitional care}

Patients not able to tolerate full rehabilitation or who don't require a high level of nursing care can continue to be managed through Shepherd's transitional care program.

\section{Outpatient services}

Eight weeks post-discharge, most patients return for an outpatient check-up. Outpatient clinics (Figure 2) provide follow-up care, including a variety of therapies, urology, dermatology, gynecology, bracing and seating, and spasticity clinics as well as ongoing assistive technology, psychological and vocational services.

\section{Medical surgical care}

Shepherd Center also provides acute care in an inpatient unit dedicated to the medical and surgical treatment of complications secondary to the spinal cord injury: skin, bowel and bladder, respiratory or skeletal systems disorders. Orthopedic surgeons also perform spine stabilization surgery.

\section{A multi-disciplinary patient care team}

Shepherd Center's high standards require staff to be experienced in the care of patients with spinal cord and brain injury and committed to their specialty. With more than 700 employees, the staff/patient ratio averages 5.7 employees per patient. There is physician coverage 24 hours a day, 7 days a week, and medical consultations available in orthopedics, neurology, 


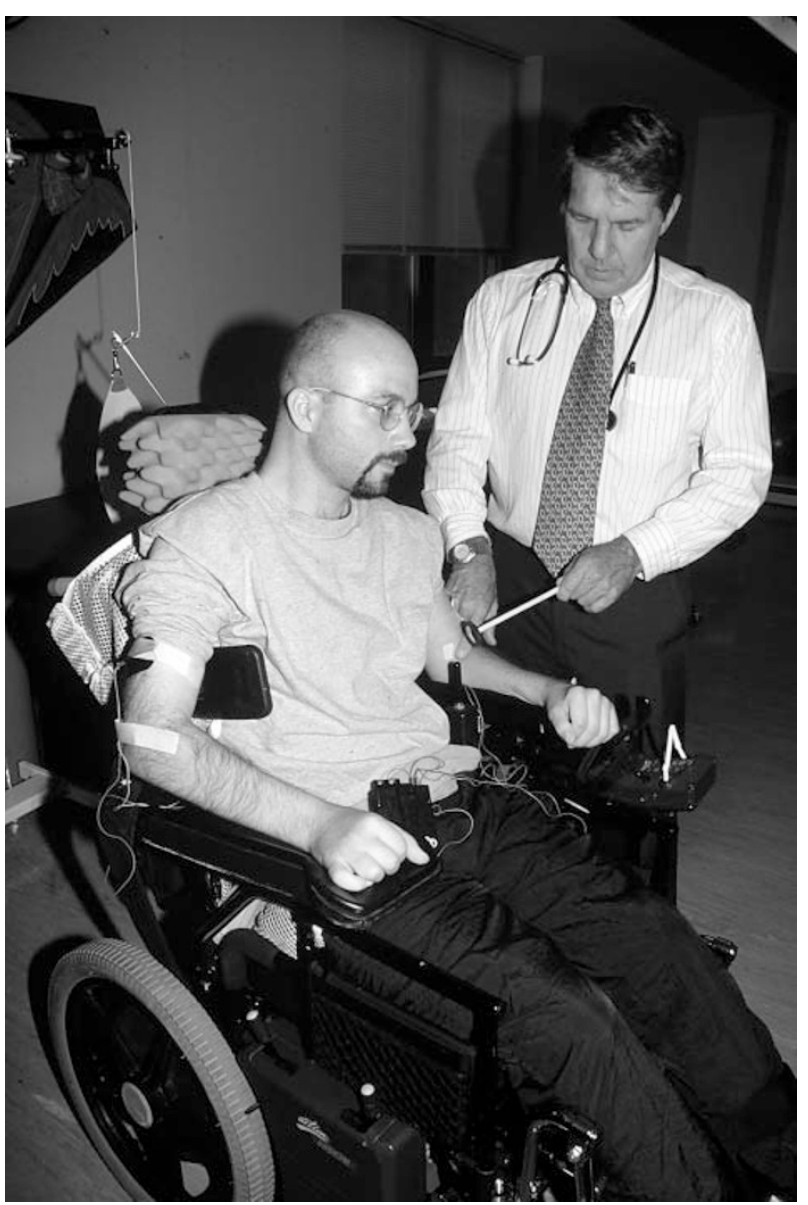

Figure 2 Since its inception in 1975, Shepherd Center's medical direction has been provided by Dr David Apple, pictured here with a patient. Dr Apple was recently honored by ASIA with a lifetime achievement award

neurosurgery, urology, plastic surgery, neuropsychology, internal medicine, gynecology, speech pathology, pulmonary medicine, general surgery and psychiatry.

\section{Clinical evaluators}

Clinical evaluators go on-site to assess patients' potential functional goals, discharge plans and funding sources prior to admission, setting in motion the initial care plan and expediting a well-informed, smooth transfer.

\section{Internal case managers}

Internal case managers lead the health care team, guiding and coordinating the delivery of services, and ensuring that goals are being met. Case managers are also the contact point for families and for insurance companies or other payors, keeping them informed of the patients' progress and making sure available financial resources are used efficiently.

\section{The team}

Managing the patient's progress is a team led by the physician, an orthopedist or physiatrist with years of experience in treating SCI and ABI patients. The team meets weekly to evaluate the patients' progress toward pre-determined goals. Team members also include a rehabilitation nurse, physical therapist, thereapeutic recreation specialist, psychologist, nutritionist, and education nurse. Supplemental staff include a respiratory therapist, speech therapist, school teacher, sexuality counselor, rehabilitation technology specialist, equipment specialist, peer supporter, chaplain and vocational counselor.

\section{Equipment}

Georgia Wheelchairs, a durable medical equipment company, is a subsidiary of Shepherd Center. At Shepherd, providing proper equipment for patients means having an on-site inventory of more than 200 chairs and a seating clinic with rehabilitation technologists to evaluate patient needs and modify chairs to fit the individual.

\section{Comprehensive services}

\section{Counseling}

Individual, group, and family counseling is provided by licensed counselors, psychologists and neuropsychologists to give patients and families a solid start in adapting to the catastrophic illness or injury and setting psychosocial goals.

\section{Nutrition}

Dietitians evaluate patients' nutritional needs and teach them how to keep healthy, energetic and strong by following a good diet.

\section{Respiratory therapy}

Among the top 18 model spinal cord injury centers in the country, less than five specialize in high quadriplegia. A key consideration in their treatment is respiratory therapy. Shepherd's respiratory therapists work aggressively with patients who are ventilator assisted to help wean them off mechanical ventilators, with a success rate of $70 \%$ over the past decade. Shepherd's ventilator program features a medical team with board certification in internal medicine, pulmonology, and intensive care who have access to the most modern equipment and techniques in ventilator care.

\section{Therapies}

Physical therapists evaluate patients for muscle strength, sensation and balance and initiate an exercise program to strengthen muscles and relearn skills. Occupational therapists evaluate strength and feeling 
in the upper body and work to strengthen those muscles used for eating, grooming, dressing, driving and job performance. Speech therapists help patients overcome hearing, speaking, chewing and swallowing or memory/concentration difficulties, sometimes using assistive techniques or special equipment.

Therapeutic recreation specialists assist patients in becoming independent and mobile in all areas of the community and teach them adaptations that allow them to pursue new or formerly enjoyed leisure activities.

\section{Urology services}

Nurses and nurse educators teach patients and family members how to prevent damage to digestive and urinary systems. Urologists make weekly rounds with physicians to answer individual questions. After discharge, Shepherd's Urology Clinic assists in preventive and follow-up care and provides male fertility services for men who want to have children.

\section{The extras}

Shepherd Center equates rehabilitation with community reintegration: giving every patient the opportunity to return to work, to school, to leisure activities and a productive lifestyle.

\section{Assistive technology}

Shepherd capitalizes on strides in space age technology to help people who have limited mobility to take greater control of their lives and their environment. By customizing wheelchairs, seating systems, electronic equipment and computer assisted technology, Shepherd's assistive technology professionals help individuals achieve maximum independence. Therapists and rehab technology specialists together recommend chairs that are safe, comfortable and appropriate. Virtually all electrical devices in the home - phones, call systems, lights, applicances, televisions, and stereos - can be operated by patients with minimal movement. Computer systems and accessories open up a new world of communication. A home accessibility team helps families determine modifications needed to return patients to their living quarters. Shepherd even has a driving program to evaluate and train patients who want to drive and to prescribe equipment for adapted cars and vans.

\section{Education}

Shepherd involves family members from the moment the patient comes to the hospital until long after they have returned home. Family members get intensive education and training, learn to become caregivers and patient advocates, receive psychological counseling, and share in peer support. Detailed care manuals are provided for patients and families alike. Individual ethnic, cultural and racial backgrounds and their accompanying beliefs and customs are taken into account in developing individualized care plans. Teachers provide schooling for young patients to ensure students can keep up with their class.

\section{Peer support}

Shepherd's Peer Support Program matches hospitalized patients with former patients who serve as role models for successful adjustment and community reintegration. Almost $90 \%$ of newly injured SCI patients are visited by one of Shepherd's 230 select peer supporters, who reside throughout Georgia and the Southeast. The program matches patients based on injury level and includes hospital and home contacts as well as educational sessions for patients and families.

\section{Vocational services}

Vocational Services has assisted hundreds of Shepherd patients meet long-term education and career goals. Services include career counseling, vocational and work evaluation, resource development, transition-to-work programs, job seeking skills, job development, and job placement. An internship program matches patients with employers for 90-days, giving them work experience and exposure to career options. Shepherd brings together employers, job seekers, and community resource and service providers to provide networking opportunities.

\section{Therapeutic recreation}

Shepherd has found that recreation is one of the best ways to encourage patients to return to pre-injury lifestyle. Recreational activity also has serious rehabilitation benefits: increasing self esteem, improving health, and reducing post-injury complications, recurring health problems and rehospitalizations. TR helps patients explore leisure lifestyles and interests and plan specific goals. Specialists focus on wheelchair sports, cultural arts, outdoor activities, horticulture and aquatics. In sports, Shepherd sponsors teams for track and field, quad rugby, basketball, fencing, bass fishing, and offers instruction in scuba diving. These are facilitated by day, evening and weekend community reintegration outings and mobility training and skills development with adaptations in equipment or techniques appropriate to the individual. Cultural art classes include painting, pottery, drama, music, woodworking and photography. Hunting trips, fishing competitions, and camping are offered to patients through the outdoor activities program. The hospital also has its own accessible greenhouse and two raised garden beds for people with balance problems and wheelchair users. Adapted tools, such as hand tools and riding mowers, are available.

The ProMotion health and wellness center at Shepherd is a unique resource, providing a fully 


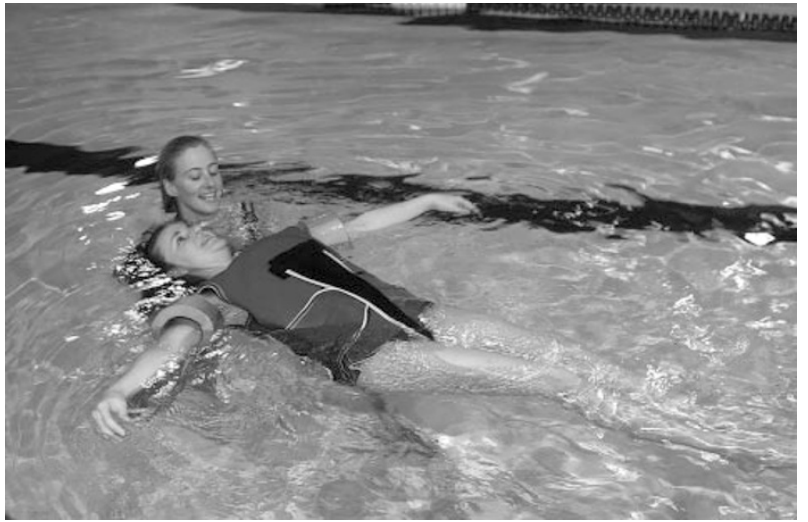

Figure 3 Shepherd's ProMotion Fitness Center has a 25yard fully accessible pool where patients with arthritis, multiple sclerosis, spinal cord injury, brain injury and other neuromuscular disorders enjoy exercise programs

accessible 25-yard pool (Figure 3) with ramps and powered lifts, a full-court gymnasium, an indoor track and a strength and cardiovascular training weight room, including private lockers, roll-in showers and dressing rooms.

\section{Commitment to people with disabilities}

\section{Experience}

From its very beginnings in 1975, Shepherd Center has set high standards, maintained a steady census, and earned a positive reputation. By focusing on very specialized rehabilitation, the hospital has developed a unique expertise in the field of catastrophic care. In only 22 years, Shepherd Center has become a nationally respected model for rehabilitation. But at its heart, it is a facility founded by a patient and his family who personally experienced catastrophic injury. The Shepherd family's support and determination is one of the many reasons that Shepherd Center remains focused on its mission of helping people with disabilities to achieve their goals with hope, dignity and independence.

\section{Advocacy}

Since 1987, Shepherd has had a coordinator for advocacy and community support and a program that builds networks to provide information and opportunities for action to individuals who want to improve accessibility, foster independent living, and encourage full community reintegration.

For 15 years, the hospital has supported the wheelchair division of one of the nation's largest $10 \mathrm{~K}$ runs, the Peachtree Road Race. The hospital also hosts an annual Adventure Skills Workshop teaching participants to scuba dive, water-ski, ride all-terrain vehicles, fish, or shoot, and enjoy outdoor activities and camaraderie.
With a history of support for wheelchair athletes, Shepherd took the lead in presenting a successful bid for the 1996 Atlanta Paralympic Games and offered initial financial support, office space and staffing. The first Paralympics held fully in the United States and funded by corporate sponsorships brought more than 100 nations and 4000 athletes here and left a legacy of financial support for athletes with disabilities, trained volunteers to support such sport programs, improved accessibility for people with disabilities, increased involvement of the business community, and a change of attitude towards people with disabilities.

\section{The Noble Learning Resource Center}

Reference and text books, journals, professional magazines, video and audio tapes, CDs and on-line information on spinal cord injury and disease, acquired brain injury, multiple sclerosis and other neuromuscular disorders, post-polio and urological disorders are available in Shepherd's fully accessible Noble Learning Resource Center. Open to patients, families, staff, and the community the library is a quiet place to read as well as a resource for literature searches, interlibrary loans or internet access.

\section{The prevention program: Think First}

Students in Atlanta's middle and high schools learn how to prevent brain and spinal cord injuries through Shepherd Center's injury prevention program. Think First was developed by the American Association of Neurological Surgeons and the Congress of Neurological Surgeons, teaching safety precautions in diving, driving, sports, hunting and climbing ladders. Hunter safety cards and driving safety cards for students attending graduation parties are provided as a community service.

\section{Education}

Shepherd Center fulfills an educational commitment by offering training for many professional programs. Residents in orthopedics and physiatry come through training programs at Emory University School of Medicine and Georgia Baptist Medical Center and physician assistants from Emory and the Medical College of Georgia. The majority of the training programs are in nursing, physical therapy, occupational therapy, and respiratory therapy. However, Shepherd also provides training for professionals in counseling, speech therapy and health information systems (medical records). In addition to affiliation with respected Georgia colleges, Shepherd provides training programs for Boston University in Massachusetts, Clemson University in South Carolina, Creighton University in Nebraska, and universities in Alabama, Connecticut, Florida, Illiniois, Indiana, Kentucky, Louisiana, Michigan, Missouri, Mississippi, North Carolina, Tennessee, Texas and Virginia. 


\section{The Crawford Research Institute}

Research at the Virginia C. Crawford Research Institute at Shepherd Center focuses on the study of the long-term effectiveness and benefits of rehabilitation, new and cost-effective ways to delivery services, and new treatments, drugs, devices and surgical procedures.
Recent projects include studies on: telemedicine, muscle and bone deterioration following SCI; an experimental method of improving male fertility after SCI; the relationship of race and gender to rehabilitation after SCI; prevention of secondary complications; employment after SCI; the health and quality of life of people aging with SCI; and neurological complications following posterior cervical wiring. 